\title{
Symptoms of the oral cavity and their association with local microbiological and clinical findings-a prospective survey in palliative care
}

\author{
Bernd Alt-Epping • Ramtin Kordestani Nejad • \\ Klaus Jung • Uwe Groß • Friedemann Nauck
}

Received: 13 August 2010 / Accepted: 2 February 2011 / Published online: 19 February 2011

(C) The Author(s) 2011. This article is published with open access at Springerlink.com

\begin{abstract}
Purpose Symptoms of the oral cavity clearly encompass more than radiation or chemotherapy-induced mucositis. Still, the burden of oral symptoms in palliative care has hardly been addressed directly, and considerations towards underlying disease processes have often been extrapolated from oncology patients.

Methods We therefore conducted a prospective explorative survey with pilot character on patients on a specialized palliative care unit, describing symptom pattern (self assessment), clinical signs, bacteriological, mycological and virological findings, and correlating features of clinical history.

Results Taste disturbances, dry mouth and the presence of Candida were found to be the most prevalent and correlating items. The broad spectrum of further symptoms, signs and findings did not show relevant correlations and did not permit any unilateral causal attributions.
\end{abstract}

Bernd Alt-Epping and Ramtin Kordestani Nejad contributed equally to the manuscript.

B. Alt-Epping $(\bowtie) \cdot$ R. K. Nejad $\cdot$ F. Nauck

Department of Palliative Medicine,

University Medical Center, Göttingen,

37099 Göttingen, Germany

e-mail: bernd.alt-epping@med.uni-goettingen.de

K. Jung

Department of Medical Statistics,

University Medical Center, Göttingen,

37099 Göttingen, Germany

U. Groß

Department of Medical Microbiology,

University Medical Center, Göttingen,

37099 Göttingen, Germany
Conclusions Apart from the described focus on Candida colonisation/infections, a symptom-guided polypragmatic approach therefore seems to be justifiable for patients with oral symptoms in palliative care.

Keywords Palliative care - Oral symptoms - Taste disturbances $\cdot$ Dry mouth $\cdot$ Candida

\section{Introduction}

As compared to "typical" symptoms and clinical problems in palliative care like pain, dyspnoea and nausea [1], symptoms of the oral cavity have drawn little attention up to date. In a large national survey (the German Hospice and Palliative Care Evaluation), the prevalence of oral symptoms like dysphagia or dry mouth have been rated by staff to be $3.5 \%$ or even below [2]. The multifaceted (medical, nursing or psychosocial) aspects of oral care in palliative care patients, underlined also in this survey, and day-to-day clinical practice suggest a broader symptom spectrum and a higher subjective relevance than epidemiological studies might imply.

Background information concerning possible causes of oral symptoms, their underlying clinical or microbiological features or standardised treatment concepts are described predominantly for oncological patients, receiving medical anticancer therapy or radiation therapy. For this group of patients, aspects of treatment-related oral and mucosal toxicity (i.e. mucositis, treatment-related xerostomy) have been described in detail [3,4], and well-proven treatment recommendations exist (Multinational Association of Supportive Care in Cancer; [5]).

In advanced cancer and in palliative care, though, oral complications and the underlying causes for oral symptoms 
and their correlation to the clinical course, drug intake, local findings and local microbiological features have been addressed to a much lesser extent until recently $[6,7]$

We therefore performed the following pilot study in order to assess the subjective symptom burden, the prevalences of clinical and microbiological oral findings and to generate hypotheses about possible underlying correlations. Thereby, we intended to contribute on defining causal relations between subjective symptoms and objective findings and resulting therapeutic implications specifically for the group of palliative care patients.

\section{Methods}

Study design and patients

We conducted a prospective single center survey, focussing on all inpatients consecutively admitted on our palliative care unit. Exclusion criteria comprised inability to take part due to the underlying clinical status, or unwillingness to give informed consent.

The survey was conducted in a five-step approach: after written information and informed consent, a thorough history was taken, including medications and prior or concurrent anticancer therapies. Patients were requested to assess their general symptom burden by using the four-step Minimal Documentation Scale (Midos) [8] and the intensity of specific oral symptoms using numerical rating scales (NRS). A physical examination of the oral cavity was performed by an approved dentist. Documentation comprised semiquantitative item scales, multiple choice and open questions, and the decayed/missing/filled teeth (DMF-T) score as a rough measure on prevalence of caries (www.whocollab.od.mah. se/expl/orhdmft.html). A mouth swab (Transystem ${ }^{\circledR}$ ) was taken from buccal mucosa, the palatal ring and tongue and sent for semiquantitative bacteriological and mycological culture, followed by a mouth wash (aqua dest. $10 \mathrm{ml}$ ) for quantitative herpes simplex virus (HSV) DNA testing, using polymerase chain reaction (PCR) technique. The dental status, the prevalence of caries (using DMF-T index) and dental hygiene were assessed by clinical inspection without staining; dental hygiene was described semiquantitatively (good-moderate-poor). A previous academic survey on oral health of a healthy working population $(n=100)$ served as a control group with respect to microbiological findings. These (unpublished) data were intentionally not matched with the study group with respect to age, health status or socio-economic background, and were collected by the Institute for Medical Microbiology, Göttingen in 2006.

The survey was approved by the local ethics authorities.
Statistical methods

The joint occurrence of symptoms and findings was studied by Fisher's exact test and further described by odds ratios. The global significance level was set to $\alpha=5 \%$ and due to the testing of multiple endpoints corrected according to the method of Bonferroni. Statistical analyses were carried out with the free software $\mathrm{R}$ (version 2.8, www.r-project.org).

\section{Results}

Patients

Within 8 months, 171 consecutive inpatients were surveyed and 101 patients were enrolled in this study (patients not included had refused study participation or were in a severely compromised general status). Table 1 lists the demographic characteristics of the study population. The most prevalent underlying disease entity was cancer of the gastrointestinal tract (30 patients) and bronchial carcinoma (22 patients), whilst head and neck tumours, affecting directly the oral cavity, contributed in only 2 patients. All patients suffered from cancer; there were no patients with leading non-cancer disease. Oral care was performed in an individualized (i.e. non-standardised) manner. Pain syndromes (other than pain of the oral cavity), fatigue and weakness were the most often self-reported general physical symptoms in Midos. From these general characteristics, the surveyed patient group was comparable to other palliative care patient collectives [2].

\section{Symptoms}

Self-reported symptoms (Table 2 ) comprised dry mouth ( 83 patients), taste disturbances (68 patients), dysphagia (29 patients), halitosis (26 patients) and pain of the oral cavity (4 patients). The intensity of these specific symptoms was described from quite low (NRS 0-1) or moderate (NRS 45) to quite high (NRS 9-10), reflecting the whole spectrum without main focus (Table 3).

The occurrence of specific symptoms of the oral cavity in patients taking sedative, anticholinergic or analgesic medication was found to be very high (e.g. 97\% likelihood for dry mouth in patients receiving morphine, or $81 \%$ likelihood of taste disturbances in patients taking lorazepam), but only a minority of patients with dry mouth or taste disturbances received opioids or lorazepam, owing tribute to the heterogenous and non-uniform intake of medications in the study population. A significant correlation between symptoms and drugs could therefore not be verified. 
Table 1 Patient characteristics (total $n=101$ )

\begin{tabular}{|c|c|}
\hline Parameter & Absolute numbers \\
\hline \multicolumn{2}{|l|}{ Age distribution } \\
\hline$>80$ years & 12 \\
\hline $60-80$ years & 49 \\
\hline $40-60$ years & 35 \\
\hline $20-40$ years & 5 \\
\hline$<20$ years & 0 \\
\hline \multicolumn{2}{|l|}{ Gender distribution } \\
\hline Male & 41 \\
\hline Female & 60 \\
\hline \multicolumn{2}{|l|}{ Antineoplastic therapy } \\
\hline Chemotherapy within last 6 weeks & 25 \\
\hline Radiation therapy within last 6 weeks & 15 \\
\hline None & 61 \\
\hline \multicolumn{2}{|l|}{ Underlying disease } \\
\hline Gastrointestinal tract cancer & 30 \\
\hline Bronchial carcinoma & 22 \\
\hline Breast cancer & 14 \\
\hline Gynaecological tumours & 6 \\
\hline Lymphoma/leukaemia & 6 \\
\hline Renal/urinary tract tumours & 5 \\
\hline CNS tumours & 5 \\
\hline Prostate cancer & 4 \\
\hline Skin cancer & 4 \\
\hline ENT tumours & 2 \\
\hline Cancer of unknown primary & 2 \\
\hline Sarcoma & 1 \\
\hline
\end{tabular}

Table 3 Intensity of the most often named symptoms

\begin{tabular}{lrr}
\hline & Dry mouth & Taste disturbances \\
\hline NRS 0 & 15 & 10 \\
NRS 1 & 16 & 11 \\
NRS 2 & 4 & 3 \\
NRS 3 & 2 & 2 \\
NRS 4 & 5 & 4 \\
NRS 5 & 19 & 19 \\
NRS 6 & 0 & 0 \\
NRS 7 & 1 & 1 \\
NRS 8 & 4 & 2 \\
NRS 9 & 4 & 2 \\
NRS 10 & 13 & 14 \\
Total & 83 patients & 68 patients \\
\hline
\end{tabular}

Clinical and microbiological findings

Clinical inspection revealed mainly unremarkable ageadjusted findings (DMF-T ranging from 7.5 on average in the age group 30-40 years, and 24.8 in the age group 80 years and older; Table 4 ).

Plaques not resembling Candida/thrush were the most prevalent finding on clinical examination (Table 2). An abnormal bacterial spectrum, defined as quantitative alterations of otherwise physiologically colonizing bacteria of the oral cavity and/or evidence of non-physiological bacteria, was found in 34 patients. In 10 patients, two distinct species were prominent; 1 patient showed a cluster of three pathogens (Enterobacter cloacae/Pseudomonas
Table 2 Absolute frequency of symptoms, according to clinical and microbiological findings $(n=$ 101)

\begin{tabular}{llrrrrr}
\hline Clinical/microbiological finding & \multicolumn{2}{l}{ Symptom } & & & \multirow{2}{*}{ Total } \\
\cline { 2 - 6 } & Pain & Dry mouth & Halitosis & Dysphagia & Dysgeusia & \\
\hline Thrush (tongue) & 2 & 22 & 10 & 7 & 17 & 23 \\
Other plaques (tongue) & 4 & 70 & 21 & 26 & 58 & 81 \\
Thrush (oral mucosa) & 2 & 30 & 12 & 9 & 23 & 32 \\
Other plaques (oral mucosa) & 3 & 57 & 17 & 22 & 45 & 63 \\
Erythema (gingiva) & 3 & 54 & 20 & 18 & 44 & 62 \\
Oedema (gingiva) & 0 & 16 & 6 & 8 & 15 & 19 \\
Haematoma (gingiva) & 0 & 4 & 2 & 1 & 4 & 5 \\
Pressure sore & 0 & 14 & 5 & 3 & 10 & 15 \\
Aphtous ulceration & 1 & 12 & 8 & 5 & 12 & 14 \\
Gingiva unremarkable & 1 & 27 & 5 & 10 & 22 & 36 \\
Oral mucosa unremarkable & 1 & 22 & 4 & 8 & 20 & 32 \\
HSV PCR+ & 2 & 17 & 8 & 4 & 14 & 19 \\
Yeasts/candidiasis + & 2 & 63 & 18 & 22 & 53 & 72 \\
Abnormal bacterial milieu & 1 & 30 & 13 & 13 & 28 & 34 \\
Multiresistant bacteria & 0 & 4 & 2 & 1 & 4 & 4 \\
Total & 4 & 83 & 26 & 29 & 68 & \\
\hline & & & & & & \\
\hline
\end{tabular}


Table 4 Age-adjusted prevalence of caries and dental hygiene

\begin{tabular}{lcccc}
\hline \multirow{2}{*}{ Age } & Prevalence of caries (average DMF-T index) & \multicolumn{2}{l}{ Dental hygiene } & \\
\cline { 3 - 5 } & & "Poor" & "Moderate" & "Good" \\
\hline$<30$ years $(n=1)$ & 18 & 1 & & \\
$30-40$ years $(n=4)$ & 7.5 & 1 & & 3 \\
$41-60$ years $(n=35)$ & 17.9 & 11 & 8 & 16 \\
$61-80$ years $(n=49)$ & 22.2 & 11 & 21 & 17 \\
$>80$ years $(n=12)$ & 24.8 & 6 & 2 & 4 \\
\hline
\end{tabular}

putida/Klebsiella oxytoca). Four mouth swabs revealed multiresistant bacteria $(\times 3$ methicillin-resistant Staphylococcus aureus, MRSA; $\times 1$ vancomycin-resistant enterococci, VRE).

Yeasts were found in mouth swabs of 70 patients, as compared to 37 subjects of the control group (healthy working population, Table 5). In 10 patients, two different Candida spp. were isolated (Table 6). HSV DNA was detected by PCR in 19 patients, with mean low to moderate titers.

The microbiological finding of Candida spp. was not always related to the clinical finding of overt thrush (from 72 patients with yeasts, 28 patients also showed thrush on mucosa), but in 52 of $72(61.1 \%)$ and 21 of $72(29.1 \%)$, respectively, of patients with yeasts, no thrush or even other types of plaques could be found. Patients showing plaques resembling thrush (defined as white, patchy pseudomembraneous plaques on erythematous ground) had a microbiological confirmation of Candida spp. in 20 of 23 (86.9\%, tongue) or 28 of $32(87.5 \%$, mucosa) of cases, respectively. Plaques that did not show these clinical characteristics were

Table 5 Microbiological findings in a healthy working population (historical control group, $n=100$ )

\begin{tabular}{lc}
\hline Microbiological findings & Frequency (\%) \\
\hline Candida spp. & 37 \\
Streptococcus viridans & 11 \\
Escherichia coli & 5 \\
Klebsiella pneumoniae & 5 \\
Enterococcus faecalis & 5 \\
Neisseria species & 4 \\
Staphylococcus aureus & 3 \\
Coagulase-negative staphylococci & 6 \\
Pseudomonas aeruginosa & 1 \\
Moraxella catarrhalis & 4 \\
Streptococcus pneumoniae & 3 \\
Serratia marcescens & 1 \\
Group A streptococci & 3 \\
\hline
\end{tabular}

An abnormal bacterial spectrum was defined as quantitative alterations of otherwise physiologically colonizing bacteria of the oral cavity and/ or evidence of non-physiological bacteria still associated with yeasts in microbiological culture in $74.1 \%$ (60 of 81 , tongue) or $80.9 \%$ (51 of 63 , mucosa) of cases. Other possible specifications of oral candidiasis like hyperplastic type, erythematous type or angular cheilitis were not differentiated further.

Symptoms versus clinical and microbiological findings

The most prevalent symptoms, dry mouth and taste disturbances, were reported in all clinically or microbiologically defined subgroups. The most prevalent clinical finding, plaques other than thrush, and the leading microbiological finding (yeasts in 72 of 101 patients.) was found in all subgroups defined by symptoms. Significant (or nearly significant) correlations were demonstrated in only six pairs of symptoms and findings (Table 7). However, only the correlation between dry mouth and other plaques (oral mucosa) is significant compared to a Bonferroni-adjusted significance level.

\section{Discussion}

From a clinical perspective, correlating symptoms and findings appear to be helpful if certain symptoms (e.g. dry mouth or taste disturbances) are associated with a specifically treatable finding (e.g. thrush/yeasts), or if certain clinical or microbiological findings (e.g. yeasts or altered bacterial spectrum, defined as quantitative or qualitative alterations of the physiological bacterial spectrum of the oral cavity) are associated with burdensome symptoms and therefore imply a need to react. Taking this into consideration, there are only few symptoms that positively predict the occurrence of a specifically treatable cause (Table 8),

Table 6 Presence of two Candida spp. in 10 palliative care patients

\begin{tabular}{lc}
\hline Species & Absolute numbers \\
\hline Candida albicans + Candida lambica & 1 \\
Candida albicans + Candida krusei & 2 \\
Candida albicans + Candida glabrata & 5 \\
Candida tropicalis + Candida glabrata & 2 \\
\hline
\end{tabular}


Table 7 Pairs of findings and symptoms that frequently come along with each other

\begin{tabular}{|c|c|c|c|c|}
\hline \multirow[t]{2}{*}{ Symptom } & \multirow[t]{2}{*}{ Finding } & \multicolumn{2}{|c|}{ Symptom present } & \multirow[t]{2}{*}{$p$} \\
\hline & & No & Yes & \\
\hline \multirow[t]{3}{*}{ Taste disturbances } & Presence of Candida & & & \multirow[t]{3}{*}{0.06} \\
\hline & No & $14(48 \%)$ & $15(52 \%)$ & \\
\hline & Yes & $19(26 \%)$ & $53(74 \%)$ & \\
\hline \multirow[t]{3}{*}{ Dry mouth } & Presence of Candida & & & \multirow[t]{3}{*}{0.04} \\
\hline & No & $9(31 \%)$ & $20(69 \%)$ & \\
\hline & Yes & $9(13 \%)$ & $63(87 \%)$ & \\
\hline \multirow[t]{3}{*}{ Taste disturbances } & Abnormal bacterial spectrum & & & \multirow[t]{3}{*}{0.03} \\
\hline & No & $27(40 \%)$ & $40(60 \%)$ & \\
\hline & Yes & $6(18 \%)$ & $28(82 \%)$ & \\
\hline \multirow[t]{3}{*}{ Dry mouth } & Thrush (oral mucosa) & & & \multirow[t]{3}{*}{0.05} \\
\hline & No & $16(23 \%)$ & $53(77 \%)$ & \\
\hline & Yes & $2(6 \%)$ & $30(94 \%)$ & \\
\hline \multirow[t]{3}{*}{ Dry mouth } & Other plaques (tongue) & & & \multirow[t]{3}{*}{$<0.05$} \\
\hline & No & $7(35 \%)$ & $13(65 \%)$ & \\
\hline & Yes & $11(14 \%)$ & $70(86 \%)$ & \\
\hline \multirow[t]{3}{*}{ Dry mouth } & Other plaques (oral mucosa) & & & \multirow[t]{3}{*}{$<0.01$} \\
\hline & No & $12(32 \%)$ & $26(68 \%)$ & \\
\hline & Yes & $6(10 \%)$ & $57(90 \%)$ & \\
\hline
\end{tabular}

Only the correlation between dry mouth and other plaques (oral mucosa) is significant compared to a Bonferroniadjusted significance level
The high incidence of Candida spp. as the leading microbiological finding denotes the most remarkable result of the study, showing quite different microbiological characteristics in the given palliative care population, almost doubling regular prevalence in healthy subjects. This finding stands out even more, as the bacterial environment of the oral cavity was found to be not relevantly altered compared to a healthy working population, with the exception of an increased prevalence of multiresistant bacteria, obviously related to preceding hospitalisations.

Also, a relatively low number of our patients (19) have been positively tested for herpes simplex virus by PCR. Their spectrum of symptoms did not differ from other patient subgroups; especially, there was no increase in oral pain, as might have been suggested. Only two of the four patients addressing pain as a relevant symptom of the oral cavity had positive HSV PCR results.

A number of factors like thrush, yeasts, herpes simplex virus, anticholinergic drugs, benzodiazepines and opioids etc. went along with the perception of dry mouth, less often with the perception of altered taste. Significant correlations, though, predominantly involved the criteria of dry mouth,

Table 8 Treatable findings accompanying specific symptoms

\begin{tabular}{llll}
\hline If symptom & Then finding & In $x \%$ of cases & Odds ratio, 95\% confidence interval \\
\hline Taste disturbances & Presence of Candida & $78 \%$ & $2.58,[0.96,6.99]$ \\
Dry mouth & Presence of Candida & $76 \%$ & $3.11,[0.95,10.25]$ \\
\hline
\end{tabular}

The last column gives the odds ratio of patients with the listed symptom having the finding compared to patients without the symptom 
Table 9 Symptoms accompanying specific clinical or microbiological findings

\begin{tabular}{llll}
\hline If finding & Then symptom & In $x \%$ of cases & Odds ratio, 95\% confidence interval \\
\hline Presence of Candida & Dry mouth & $88 \%$ & $3.11,[0.95,10.25]$ \\
Presence of Candida & Taste disturbances & $74 \%$ & $2.58,[0.96,6.99]$ \\
Abnormal bacterial spectrum & Taste disturbances & $82 \%$ & $3.12,[1.07,10.47]$ \\
Thrush (oral mucosa) & Dry mouth & $94 \%$ & $4.47,[0.95,42.73]$ \\
Other plaques tongue & Dry mouth & $86 \%$ & $3.37,[0.93,11.82]$ \\
Other plaques mucosa & Dry mouth & $90 \%$ & $4.31,[1.33,15.65]$ \\
\hline
\end{tabular}

taste disturbances or yeasts/thrush respectively. As many conditions lead to a stereotype symptom pattern, clear causal relations, as attributed for instance to the role of anticholinergic drugs or opioids, can hardly be extrapolated in a complex palliative care setting.

This study allows a description of clinical and pathobiological associations rather than causal relations. The remarkably high prevalence of Candida spp. in the oral cavity may well be a causative factor for oral symptoms like dry mouth or taste disturbances (and therefore a possible target for empirical antifungal treatment). At the other hand, xerostomia and taste disturbances may rather be caused by salivary gland dysfunction (suggesting the substitution of saliva or therapeutic interventions to stimulate the salivary glands), that is followed by Candida infection. Therefore, future research agenda should involve efforts for a better understanding of the complex pathophysiological processes within the oral cavity of palliative care patients, including the potential role of cancer itself and its metabolic and immunological impact.

Nevertheless, even the mere description of clinical and pathobiological associations might be clinically useful: if a patient complains about dry mouth, the clinical alertness for associated Candida infection should be high.

This study faces several more interpretative limitations

Besides the above-named microbiological results, oral health aspects could not be investigated in all details in this pilot survey on severely ill patients with incurable advanced cancer. The semiquantitative assessment of dental hygiene and the assessment of caries by the DMF-T index suggest a rather unremarkable standard of oral hygiene and a relatively uncompromised condition of teeth in the surveyed patient population, but these items do not encompass all aspects of oral health. Especially, the gingival condition and a more detailed look on dental hygiene, as well as their possible correlations to symptoms and microbiological outcomes should be a focus in further studies. Also, patients in severely affected general condition had to be excluded from further evaluation; therefore, the prevalence of oral complications might have been even higher.

The pattern of symptom intensity (Table 4) suggests practical problems in using the numerical rating scales and a selection bias towards mean and extreme values.

The pilot character of this study in general opposes multiple testing for significances for statistical reasons. The significances that have been calculated despite this methodological obstacle have to be rather interpreted as relevant correlations, and require prospective confirmation.

A number of aspects concerning the clinical relevance of several microbiological findings remain under further debate

In four patients, multiresistant bacteria like MRSA or VRE were diagnosed incidentally. This sheds light on hygiene standards or isolation policies, not seldomly conflicting palliative care priorities.

The "clusters of pathogens", multiple quantitative and qualitative bacterial alterations found in 11 patients focus on the aspects of bacterial colonisation versus infection, and deserve further consideration. The findings on yeasts and thrush give a first glimpse on the interdependency of microbiological and clinical findings and subjective relevance. Conclusions concerning changes over time, like the likelihood for progression from a subclinical, microbiologically confirmed yeast colonisation to overt thrush, require further longitudinal study.

Ten patients had two Candida spp., and the relevance of antifungal (especially azoles) drug resistance may be highlighted by this finding, even if antifungal resistance testing was not performed.

The microbiological criteria of an abnormal bacterial spectrum that was found in 34 patients and showed to be comparable to control group persons with respect to prevalence and leading pathogens need to be discussed. Also, anaerobic bacteria were not routinely screened due to technical colonisation reasons, and the microbiological findings that served as control group were not collected for this purpose. 
Furthermore, the survey faces limitations with respect to missing details about grading of symptoms and signs, about previously taken antibiotics or antimycotics, food intake on the day of clinical examination and mouth swab/mouth wash, respectively.

There are only a few preceding investigations on palliative care patients that also focus on oral symptoms (e.g. [12]) or the prevalence of oral infections/colonisations (e.g. [13]). In these studies, the symptom burden has been found to be up to $77 \%$ (dry mouth [14]), and the prevalence of microbiologically proven candidiasis was found to be $26 \%$. A recent survey from Norway [15] showed symptom prevalences and clinical findings similar to our patient cohort, except from the much higher prevalence of oral pain that might be attributed to anticancer treatment-related mucositis.

In these studies, it was not intended to detect possible associations between subjective local symptoms and clinical or microbiological findings, though. In order to extend therapeutic options in oral palliative care, the preceding and present study contribute to a better understanding of a comprehensive therapeutic approach.

\section{Conclusion}

The burden of symptoms of the oral cavity is not routinely assessed in palliative care and has obviously been underestimated in previous studies. As many of the described clinical, pharmacological or microbiological conditions are associated with a stereotype pattern of oral symptoms, a clinical practice of unilateral allegations (e.g. towards a negative role of opioids) cannot be justified. Instead, an increased awareness of oral problems, a symptom-guided assessment and a therapeutic approach that prioritises the patients' symptoms over diagnostic findings in therapeutic decision making, seems to be more promising from a palliative care perspective.

Acknowledgments We are most grateful to Prof. Dr. H. Eiffert (Department of Medical Microbiology), Prof. Dr. G. Hunsmann (Department of Virology), Prof. Dr. H. Schliephake (Department of Oral and Maxillofacial Surgery), all three from of the University Medical Center Göttingen, for the helpful discussions and support, and to the patients on our palliative care unit and to all staff members that made this pilot project possible.

Disclosures None
Conflicts of interest The authors declare that there is no organization that sponsored the research. The authors have full control of all primary data and they agree to allow the journal to review their data if requested.

Open Access This article is distributed under the terms of the Creative Commons Attribution Noncommercial License which permits any noncommercial use, distribution, and reproduction in any medium, provided the original author(s) and source are credited.

\section{References}

1. Higginson IJ (1997) Health care needs assessment: palliative and terminal care. In: Stevens A, Raftery J (eds) Health care needs assessment. Wessex Institute of Public Health Medicine, Oxford, pp 183-260

2. Radbruch L, Nauck F et al (2003) What are the problems in palliative care? Results from a representative survey. Support Care Canc 11:442-451

3. Saadeh CE (2005) Chemotherapy- and radiotherapy-induced oral mucositis: review of preventive strategies and treatment. Pharmacotherapy 25(4):540-554

4. Sweeney MP, Bagg J, Baxter WP, Aitchison TC (1997) Clinical trial of a mucin-containing oral spray for treatment of xerostomia in hospice patients. Palliat Med 11(3):225-232

5. Keefe DM, Schubert DM, Elting LS, Sonis ST, Epstein JB, RaberDurlacher JE, Migliorati CA, McGuire DB, Hutchins RD, Peterson DE (2007) Mucositis study section of the multinational association of supportive care in cancer and the international society for oral oncology. Updated clinical practice guidelines for the prevention and treatment of mucositis. Cancer 109(5):820-831

6. Davies A, Finlay I (eds) (2005) Oral care in advanced disease. Oxford University Press, Oxford

7. Davies A, Epstein J (eds) (2010) Oral complications of cancer and its management. Oxford University Press, Oxford

8. Radbruch L, Sabatowski R, Loick G et al (2000) MIDOSvalidation of a minimal documentation system for palliative medicine. Schmerz 14(4):231-239

9. Addington-Hall J, McCarthy M (1995) Dying from cancer: results of a national population-based investigation. Palliat Med 9:295-305

10. Wiseman M (2006) The treatment of oral problems in the palliative patient. J Can Dent Assoc 72(5):453-458

11. Naidu MU, Ramana GV, Rani PU et al (2004) Chemotherapyinduced and/or radiation therapy-induced oral mucositis-complicating the treatment of cancer. Neoplasia 6(5):423-431

12. Sweeney MP, Bagg J (2000) The mouth and palliative care. Am J Hosp Palliat Care 17(2):118-124

13. Bagg J, Sweeney MP, Lewis MA, Jackson MS, Coleman D, Al MA, Baxter W, McEndrick S, McHugh S (2003) High prevalence of nonalbicans yeasts and detection of anti-fungal resistance in the oral flora of patients with advanced cancer. Palliat Med 17(6):477-481

14. Jobbins J, Bagg J, Addy $M$ et al (1992) Oral and dental disease in terminally ill cancer patients. Brit Med J 304:1612

15. Wilberg P, Hjermstad MJ, Ottesen S, Herlofson BB (2009) Oral health in advanced cancer-a neglected problem? Results from a dentist's examination. European Journal of Palliative Care/1th Congress of the European Association for Palliative Care 160 